\title{
Mary Richmond: una semblanza personal e intelectual (1861-1928)
}

\author{
Mary Richmond: a personal and intellectual biographical portrait \\ (1861-1928) \\ Brigitte BOUQuET \\ Conservatoire National des Arts et Métiers, París \\ brigitte.bouquet@cnam.fr
}

Recibido: 26/04/2011

Revisado: 05/05/2011

Aceptado: 20/06/2011

Disponible on line: $29 / 09 / 2011$

\begin{abstract}
Resumen
¡150 años!... Mary Richmond nació hace 150 años. Contemporánea de Jane Addams y líder influyente dentro de los movimientos de las organizaciones caritativas americanas, desde muy joven se comprometió con la acción social y más tarde con la investigación y la formación del Trabajo Social, y abrió el camino al acercamiento al Case Work. Raros son los países en los que el Trabajo Social no sea deudor de su influencia y su reflexión.
\end{abstract}

Palabras clave: caridad, Trabajo Social, políticas sociales, Trabajo Social de caso.

\begin{abstract}
150 years! Mary Richmond was born $\square 150$ years ago. A contemporary of Jane Addams and an influential leader within American charitable organizations, she commited herself to social action from an early age and later, to research and the formation of Social Work, and she opened the way towards Case Work. There are few countries today in which the Social Work field does not owe a debt to her influence and thought.
\end{abstract}

Keywords: charity, Social Work, social policies, Social case Work.

Referencia normalizada: Bouquet, B. (2011): «Mary Richmond: una semblanza personal e intelectual (1861-1928)». Cuadernos de Trabajo Social, 24: 13-21.

Sumario: 1. Su juventud. 2. Baltimore (1889-1900): el descubrimiento de «lo social». 3. Filadelfia (1900-1909): maestra de lo social. 4. Russel Sage Foundation (1909-1922): conceptualizadora del trabajo de caso. 4.1. Administradora y participante en las políticas sociales. 4.2. Implicada en la vida ciudadana y nacional. 4.3. Profesora e investigadora de Trabajo Social. 5. Referencias bibliográficas de Mary E. Richmond. 6. Referencias bibliográficas sobre Mary Richmond.

\section{Su juventud}

Mary E. Richmond nació el 5 de agosto de 1861 en Belleville, Illinois, durante la guerra de Secesión, en la que su padre se convirtió en artillero para poder disponer de un buen sueldo. Dos años más tarde sus padres se volvieron al antiguo barrio obrero de su ciudad natal, Baltimore, donde Mary Richmond creció. Segunda de cuatro hijos, fue la única que sobrevivió. $\mathrm{Su}$ madre falleció muy joven a causa de la tuberculosis, cuando ella apenas tenía cuatro años ${ }^{1}$. La educación corrió a cargo de su abuela materna y dos tías. Ambas pertenecían a una clase social poco acomodada, pero muy abierta. Su abuela alquilaba habitaciones a buen precio en su casa, lo que les permitía relacionarse con personas muy diversas. Su abuela era sufragista radical y se interesaba por el espiritualismo. Mary E. Richmond escuchaba en ese ambiente animadas discusiones

\footnotetext{
${ }^{1}$ Su padre se volvió a casar, se ocupó poco de ella, murió cuando tenía siete años.
} 
sobre el sufragio femenino, los problemas raciales, el sacrificio de los animales, las creencias políticas y sociales y la espiritualidad. Era muy aficionada a la lectura, lo que le ayudó a formarse sus ideas y elaborar sus propios argumentos, y a adquirir así un pensamiento crítico y una actitud benévola hacía los pobres, los necesitados y los discapacitados. Se inclinaba por las ideas liberales, tenía un espíritu incisivo, una gran capacidad para examinar críticamente las ideas que se planteaban, y mucho sentido del humor.

$\mathrm{Su}$ familia no era partidaria de los métodos educativos de enseñanza de aquella época. Mary Richmond no asistió a ningún colegio público hasta los once años. Sin embargo aprendió a leer muy joven, y sin apenas vigilancia leía apasionadamente todo lo que caía en sus manos, entre otros a Charles Dickens y a María Edgeworth ${ }^{2}$.

En general no recibió una educación muy esmerada, fue autodidacta en gran medida, pero como ella misma indicaba, su «educación fue continua». El haberse quedado como niña única y su tardío ingreso en la escuela le causaron dificultades de adaptación para la vida en grupo, lo que no le impidió, sin embargo, convertirse a menudo en leader en los juegos y las actividades. Tras haber obtenido el diploma de la escuela primaria, a los trece años entró en la secundaria, en la Eastern High School ${ }^{3}$ para niñas. Creó un club de literatura clásica con el apoyo de un profesor muy apreciado por las alumnas y se interesaba por que cada miembro del mismo participase estudiando algunos artículos, a menudo complicados, pero de libre elección. Uno de sus profesores recordaba más adelante: «incluso en edad precoz para ser ya diplomada, era excepcional por sus dotes intelectuales, por su gracioso estilo, sus elevadas ideas, y su humanidad $»^{4}$ (citado en Pumphrey Muriel, 1961).

En 1878, a los diecisiete años y ya diplomada, Mary Richmond trabajó durante unas semanas en una fábrica y más tarde se reunió con su tía, que trabajaba en una editorial en Nueva York. Encontró trabajo como secretaria y desempeñó también las funciones de correctora y maquetista. Ambas compartían una habitación amueblada y largas jornadas de trabajo, a menudo de 12 a 14 horas, Richmond aprendió taquigrafía; pero su tía se enfermó y regresaron a Baltimore, donde se quedará hasta el fin de sus días, dependiendo económicamente de su sobrina que debía desenvolverse en un entorno que le era extraño. Sola, casi sin amigos, obligada a hacer frente a esta nueva situación y con pocos recursos, y aquejada por un estado de desnutrición, Mary Richmond intentó sobreponerse a la soledad de la manera mejor posible: asistiendo a las conferencias de la Cooper Union. Hacía 1886 ingresó en la Iglesia unitaria $^{5}$ y comenzó a participar activamente en las veladas para jóvenes adultos. Ella dirá que fue el periodo más amargo de su vida: «no se puede estar tan sola en una gran ciudad, tenía ansias como todas las chicas que se sienten solas, de afecto y de amigos» ${ }^{6}$.

Desde pequeña Mary Richmond vivía con la idea de que moriría de tuberculosis como sus padres. Temerosa de que pudiera padecer esta enfermedad, decidió consultar a uno de los mejores especialistas de Nueva York, previniéndole de que no podía pagarle sus honorarios. Al considerar éste que su estado era grave, le ordenó que dejase el trabajo y se fuera a des-

2 Charles Dickens, romancero muy conocido; María Edgeworth, 1767-1849, consagró su talento a la educación de la infancia y a la moralización del pueblo, compuso pequeñas obras, la mayor parte de las mismas fueron muy populares.

3 En esta época, era escaso el porcentaje de niñas que iba a la escuela secundaria, y menos aún que se diplomaba. Sólo treinta alumnas de esta escuela obtuvieron el diploma en 1878 .

${ }^{4}$ Literalmente : even at the early age of her graduation she was unusual, in mental attainments, in grace of manner, in high ideals, and the humanites. Letter to the editor after Mary Richmond had received an honorary degree. The Sun Baltimore, 8 de Julio de 1922 (cit. en Pumphrey Muriel, W. (1961). Mary Richmond, the practitioner. Review Social Casework, 42.

5 Aunque los orígenes del Unitarismo se remontan a la Reforma y de ahí proceden las tradiciones, la Iglesia unitaria en América se inspira en ellos y en un célebre sermón que el pastor William Ellery Channing pronunció en Baltimore en 1819.

${ }^{6}$ Literalmente: you cannot be so lonely as in a large city (...) I craved, just as every girl does who islonely, adfections an freinds. 
cansar al campo. Tras explicarle que le era imposible dejar de trabajar, aquél le hizo una serie de recomendaciones acerca de la dieta y el reposo y le enseñó una serie de ejercicios respiratorios. Siguiendo sus consejos, se instaló en Brooklyn y aprovechó los paseos en barco para realizar los ejercicios físicos recomendados. Su salud mejoró progresivamente, pero poco después se enfermó de paludismo, lo que le obligó a volverse a Baltimore

\section{Baltimore (1889-1900): el descubrimien- to de «lo social»}

Tras estos problemas de su salud, Mary Richmond trabajó como contable en una papelería y más tarde aceptará el empleo de contable-intendente en una pensión. Al igual que con su educación, su formación religiosa se iba construyendo de acuerdo con las circunstancias. Se negaba a aceptar cualquier dogma que no se ajustase a su inteligencia o a su sentido estético. Se acercó a la Iglesia Unitaria local, donde trabó amistades profundas y se convirtió en miembro del consejo. Muy implicada y bastante marcada por el movimiento Social Gospel, Mary Richmond fue adquiriendo seguridad en sí misma y comenzó a descubrir su vocación. Se esforzó en indagar acerca de las aplicaciones prácticas de las enseñanzas religiosas, como respuesta a los problemas sociales que acompañaban a la rápida industrialización, el urbanismo y la pobreza, y desarrolló sus competencias sociales. Ella enseñaba en «la escuela dominical», preparaba a jóvenes de su edad para el estudio a través de los textos de Shakespeare. También se abrió a la música y a la filosofía, llamando la atención su influencia y su proyección.

Se amplían sus campos de interés y se desarrollan sus capacidades, pero le agobia la monotonía de su trabajo. A los 27 años, por un anuncio del periódico The Sun de Baltimore, de 16 de diciembre de 1888 , se enteró de que la Charity Organisation Society de Baltimore estaba buscando una asistenta-tesorera:
La Charity Organisation Society busca los servicios de una mujer instruida o de un caballero de buena posición social, que conozca bien la ciudad, para ordenar las suscripciones, reclutar a nuevos miembros y dar a conocer los métodos y los fines de la asociación a todas las clases sociales de la comunidad. El salario mínimo será de $50 \$$ al mes, pudiendo aumentar, dependiendo del trabajo realizado. La respuesta del candidato debe ser manuscrita, indicando la edad, el lugar de residencia y las actividades desempeñadas anteriormente, y debe contener referencias anteriores satisfactorias, así como sus capacidades y su carácter?

Veintiuna personas postularon al puesto (algunos exprofesores, vendedores, un fabricante de cigarros, una costurera, algunos jóvenes médicos) $)^{8}$. Animada por sus amigos, aunque no conocía esta organización, se presentó una tarde lluviosa para realizar una entrevista con el presidente. Este dirá más tarde que ella tenía un aire «patéticamente joven», pero hablaba como una «sabia». En el transcurso de esta entrevista, Mary Richmond comprendió las posibilidades que estaban a punto de abrírsele. La asociación era joven y estaba endeudada, pero luchó por promover sus ideas, que eran las de combinar y desarrollar todos los recursos de beneficencia de la comunidad con el fin de aliviar la pobreza, y las de prevenir el pauperismo y el crimen y elevar el nivel social. La gran capacidad de organización de Mary Richmond, su aptitud dialogante, su ingenio y su aspiración a asumir más responsabilidades le fueron reconocidas y aceptadas.

La Chatity Organisation de Baltimore ${ }^{9}$ era la filial americana de la organización Octavia Hill, que se había establecido en el Reino Unido y que había fundado, en 1881, Daniel C. Gimman, de la Universidad John Hopkins. Fue secundada por un joven brillante, Amos G. Warner, que desarrolló la asistencia descentralizada, la pertinencia de la ayuda basada en una encuesta en profundidad. La Charity Organi-

${ }^{7}$ Literalmente: «The Charity Organisation Society desires the services of an educated lady or gentleman of good social position and well acquainted in the city to collet its subscription and extend its membership and knowledge of its methods and aims. Troughout all classes of the community».

${ }^{8}$ Falta la carta de Mary Richmond, probablemente arrasada en un incendio que destruyó toda la documentación administrativa de la agencia.

${ }^{9}$ Los archivos de la Russell Sage Foundation fueron donados a los Archivos Rockefeller en 1985. 
sation Society se proponía, como objetivos, combatir las causas de la pobreza y de la corrupción política y promover la cooperación entre las organizaciones sociales y los individuos, estableciendo una relación democrática, un puente entre los privilegiados y los desfavorecidos, entre los ricos y los pobres, teniendo en cuenta particularmente la ayuda de las friendly visitors. Esta concepción la asumió Mary Richmond con entusiasmo y consagrará su vida a ponerla en práctica.

Algunos meses más tarde iba a persuadir al presidente del comité ejecutivo, John Glen ${ }^{10}$, para que le permitiera convertirse en una «visitadora amigable». Tras un periodo de adaptación de una semana en Boston, bajo la dirección de Zilpha D. Smith que se convirtió en su inspiradora y consejera, Mary Richmond entró por primera vez en contacto con las visitadoras sociales que intentaron mostrarle los rudimentos esenciales de su actividad.

De vuelta a Baltimore retomó sus funciones de asistente-tesorera y se encargó de la propaganda. Su trabajo consistía en actualizar el estado de cuentas de las diferentes modalidades de suscripciones y colectas y ponerse en contacto con aquellas personas que no las deseaban renovar. Desde el principio trató de conseguir que su funcionamiento fuera más eficaz y mejor coordinado y desarrolló numerosas e ingeniosas formas de publicidad. Se decía que su encanto provocaba un cambio en numerosos indiferentes o en los contribuyentes escépticos, que se acababan convirtiendo en partidarios activos de los programas.

La necesidad de explicar el trabajo de su organización en conferencias (impartió una treintena en nueve meses) y su preocupación por las necesidades de las personas impulsaron a Mary Richmond a aprender cada vez más sobre las visitadoras voluntarias. En su actividad como «visitadora» encontró a personas necesitadas y les ayudó a mejorar su situación. Aunque el número de «casos» seguidos por Mary Richmond no se conoce, sus contemporáneos han contado en sus memorias que realizaba prácticas como voluntaria de forma esporádica y que nunca había trabajado con más de tres o cuatro familias simultáneamente. Comenzó a desarrollar sus numerosas ideas, observando que era necesario revisar el sistema de administración de la caridad fijado por la condescendencia de las damas «ricas», ya que se constataban numerosas incoherencias y una ausencia de lógica científica. Reflexionaba sobre la manera como se podían mejorar las intervenciones para ayudar a las personas necesitadas.

En la primavera de 1890 se celebró en Baltimore la National Conference of Charities and Correction, una oportunidad para Mary Richmond de encontrarse con los líderes de este movimiento. Presentó su primera comunicación; y representó también a la Young Women Christian Association (YWCA) de Baltimore en la Convención Nacional sobre el trabajo femenino en Nueva York.

El puesto de secretario general quedó vacante en 1891, John Glenn se lo ofreció, impresionado por su personalidad y por el trabajo realizado, a pesar de ser mujer y no tener estudios universitarios. Aunque a principios de los años 1890 el rol de administrador de un organismo social estaba mal definido, ella aceptó y rápidamente se familiarizó con las teorías administrativas enunciadas por Charles Loring Brace, de la New York Children's Aid Society, y por Franklin Sanborn, secretario de la National Conference of Charities and Correction $^{11}$. Reestructuró rápidamente la organización que se hallaba en situación de decadencia y la hizo funcionar eficazmente. Continuó su gran interés por las intervenciones y combinó sus cualidades de observadora y su atenta escucha a las visitadoras ${ }^{12}$ con la labor de teorización sobre ellas.

En 1985, cuando John Glenn se jubiló, se responsabilizó de todas las funciones de la

10 Abogado semijubilado y dirigente de empresa, se consagró casi a tiempo completo a los trabajos de la sociedad. Mantuvo un estrecho contacto con universitarios, muy interesado por las ideas sociales y las corrientes políticas en Europa y América.

11 Ambos vieron en ella rápidamente a una administradora, a una líder y a una innovadora.

12 En su artículo, Pumphrey Muriel describe a Mary Richmond como físicamente seductora, con ojos de un azul intenso y «un lenta sonrisa de aprecio que se manifestaba a lo largo de la conversación». Pumphrey Muriel W. (1961). Mary Richmond, the practitioner. Review Social Casework, 42. 
organización. Sus consejos eran muy buscados y escuchados. Su gestión consistía en lanzar un movimiento social mostrando las necesidades, revelando las carencias y creando nuevos modelos de intervención y una organización social para remediarlos (por ejemplo la lucha contra los tugurios y la nueva experiencia de las viviendas sociales). Contribuyó a la reforma de la legislación social, en particular con medidas tales como: la reforma sanitaria, la prohibición de la mendicidad y de la prostitución, la mejora de los locales de los hospicios, el trabajo infantil, la custodia de los niños, etc. Organizó algunos grupos de personas, como «el club de madres», en donde se debatía sobre los modos de tratar a los niños, de economía domestica, de los problemas familiares, etc. Publicó numerosos artículos.

Consciente de las lagunas que tenían las friendly visitors y los volunteers social services, Mary Richmond les proporcionó numerosos espacios de formación, les recibía en su casa en la que se desarrollaban tertulias nocturnas de lectura y discusión. Fomentó la creación de bibliotecas en las organizaciones locales. En la Conferencia Nacional de Organizaciones de Beneficencia de Toronto, en 1897, presentó un estimulante informe en el que proponía la formación y la creación de escuelas de servicio social y, en 1898-1899, contribuyó a la creación de la New York School of Applied Philanthropie, la primera escuela de Trabajo Social de Nueva York donde enseñará.

En 1899, Mary Richmond publicó Friendly Visiting Among the Poor. A Handbook for Charity Workers. Este opúsculo, concebido para quienes comenzaban a trabajar en la beneficencia, es una compilación de lo que creía útil en una sesión de formación y sigue siendo una de las mejores descripciones del trabajo de las visitas a domicilio realizado en este periodo. Subraya la importancia de la intervención de ayuda a la persona dentro del contexto de su grupo y de sus relaciones familiares, y de la función esencial de llevarla a cabo con la ayuda mutua y los recursos comunitarios para lograr así desarrollar un bienestar y un futuro para las familias.

\section{Filadelfia (1900-1909): maestra de lo social}

En 1900 Mary Richmond aceptó la dirección de la agencia de Filadelfia donde se encontró con un modo de funcionamiento diferente. En aquella época, mientras la COS de Baltimore hacía encuestas sobre la ayuda que prestaba y recomendaba que colaboraran en la prestación de socorro también otras organizaciones, ya que no tenían fondos propios suficientes, la agencia de Filadelfia recaudaba regularmente sumas importantes para ayudas directas, pero concedía menor importancia a la rehabilitación económica y social. Mary Richmond, sin abandonar su interés por el desarrollo de la fílosofía en las intervenciones, se vio obligada a prestar atención prioritaria a los problemas de la administración y de recaudación de fondos.

Seguía trabajando activamente en la formulación de leyes, enseñaba en los colegios de formación social creados en Boston, Nueva York y Filadelfia, y seguía escribiendo: en 1907 publicó The God Neighbor in the Modern City. Se convirtió en redactora de la revista Charities, creada por la agencia y cuyo primer proyecto fue publicar una serie de pequeños ensayos sobre diversos aspectos de la encuesta social, realizados por los trabajadores sociales más conocidos.

Mary Richmond viajó sólo una vez a Europa, en 1903, fue con unos amigos a Gran Bretaña y Escocia, y combinó las actividades culturales con el estudio del Trabajo Social: se reunió con trabajadores sociales, visitó los settlements houses, inspeccionó los talleres y mantuvo reuniones en los comités de distrito de la London Charity Organisation Society.

Tras nueve años de permanencia en Filadelfia le fue reconocida su autoridad, aunque sus intereses estaban más centrados en la investigación, la enseñanza y la escritura.

\section{Russell Sage Foundation (1909-1922): conceptualizadora del trabajo de caso}

Desde la creación de la Russell Sage Foundation $^{13}$, en Nueva York en abril de 1907, Mary Richmond sintió un gran interés por esta institución. La Fundación tenía como objetivo consagrarse a la investigación social en los cam-

${ }^{13}$ La Fundación se creó gracias a un donativo de diez millones de dólares de Margaret Olivia Slocum Sage, segunda esposa y viuda de Russell Sage, administrador de los ferrocarriles y financiero. 
pos de la inmigración, la cultura, la religión, la educación, las cuestiones económicas y sociales y el mercado de trabajo, persiguiendo «la mejora de las condiciones sociales y de vida en los Estados Unidos»».

En 1909, la Russell Sage Foundation le solicitaba a Mary Richmond que fuera la directora vitalicia del departamento de Organisation Charity. Este puesto le permitía ejercer una gran influencia como administradora y como investigadora, y desarrollar la práctica, a la vez que ejercía también de profesora.

\subsection{Administradora $y$ participante en las políticas sociales}

Como administradora de la Russell Sage Foundation, participó en el esfuerzo que realizaba la organización y en la cooperación que se estableció entre distintas asociaciones de beneficencia, para asegurar un reparto más eficaz y humano de los recursos destinados a los pobres. También la nombraron administradora de numerosos comités.

Ella cambió el nombre de la conferencia «Charities and Correction» por el de «National Conference». En la National Conference de 1910 presentó un informe sobre la interdependencia de los movimientos sociales (67 organizaciones sociales, de las cuales 39 se habían fundado en la década anterior), y la posible contribución de los trabajadores sociales a las campañas nacionales. Proponía que quienes se convirtieran en agentes locales de estos movimientos, habían de encargarse también, a nivel local, de la información y de la coordinación de las distintas actividades, a fin de que «consciente y deliberadamente estén en el punto de intersección de cada uno de los movimientos nacionales (...) un medio para reforzar el conjunto del tejido social».

A partir de su reflexión sobre el Trabajo Social concluía que las intervenciones sociales no tendrían éxito a largo plazo, si los trabajadores sociales no intervenían con las familias y los individuos, así como también dentro de los programas de reforma social. Según su opinión, estos programas podrían dar un impulso a un tratamiento más eficaz de las intervenciones, si contaran con «más variedad de recursos, adaptables a situaciones particulares».

Durante los primeros años, cuando las investigaciones versaban sobre los problemas de los pobres, los ancianos y la mejora de los hospitales, Mary Richmond creía que era necesario publicar una serie de opúsculos destinados a un amplio público que comenzaba a interesarse por los problemas sociales, que debían provocar un impacto sin entrar en muchos detalles y cuya lectura fuera fácil. Se publicaron once documentos sobre temas tales como: Madres solteras, ¿Qué es la organización de caridad?, La familia inmigrante, etc.

A Mary Richmond le consultaban los compañeros que estudiaban los efectos del desempleo. Así, en la depresión de 1921, Mary van Kleeck, que tenía que presentar una comunicación sobre el paro, contactó con ella, quien le remitió una recapitulación de medidas de urgencia. Insatisfecha por la poca difusión de los trabajos sobre desempleo, implicó a Philipp Klein en una investigación que fue publicada en 1923, con el titulo The Burdedn of Unemployment.

En 1919 examinó una serie de breves artículos que trataban de las relaciones conyugales. No sospechaba que la parte de la investigación concerniente a la aplicación de las leyes del matrimonio en Estados Unidos le iba a suponer la redacción de tres libros y un opúsculo, que tardaría cinco años en publicarse: A Study of nine hunderd and eighty widows known to certain charity organization societies in 1910. Versa sobre las familias, las diferentes situaciones laborales y el tratamiento de la protección social de las viudas. En sus últimos años se interesó por la ley de la reforma del matrimonio, sobre la que reflexiona en Child Marriages (en particular sobre los aspectos psicológicos, raciales y sociales), y en una obra póstuma, Mariage and The State, escrita con Fred Sage Hall y publicada en 1929. Sus investigaciones tuvieron un gran impacto en la mejora de la legislación. No obstante Mary Richmond se alegraba vivamente de haber vuelto a centrar la práctica en el case work.

\subsection{Implicada en la vida ciudadana y nacional}

Mary Richmond había sobrevivido a dos crisis industriales: una en 1897 y la otra en 19071908; pero la Primera Guerra mundial le sometió a una dura prueba. Desde el principio de la guerra seguía atentamente los acontecimientos, simpatizaba con los objetores de concien- 
cia, estaba de acuerdo con las políticas de no intervención del presidente Wilson y temía la declaración de guerra de Estados Unidos. Sin embargo se sintió muy involucrada con la entrada en guerra, celebró la victoria y se implicó en ella. A partir del invierno de 19141915 el desempleo pesaba duramente sobre las organizaciones de caridad y sus recursos se pusieron a disposición de ellas para paliar las consecuencias de esta crisis. El departamento que dirigía organizó también una colaboración mutua con diferentes sociedades de caridad para apoyar a los parados en sus desastrosas situaciones. Fue consultada en distintas ocasiones, por ejemplo, por John Mayor Mitchel, para su estudio sobre el paro, o por la asamblea de las organizaciones femeninas de Nueva York. Participó en un comité de apoyo a las familias extranjeras albergadas en las mismas. Deseaba que todo el mundo pusiera al servicio de la nación sus capacidades, quería que el case work se pusiera al servicio de la mayoría $\mathrm{y}$, en particular, de las familias de los soldados y de los marineros. En el Servicio de Acogimiento de la Cruz Roja dirigió a los trabajadores sociales que realizaban visitas a domicilio, en las que entrevistaban a los soldados y a sus familias, para ayudarles a solucionar los problemas detectados. Cada vez eran más numerosos los médicos, psiquiatras y psicólogos que consideraban a los trabajadores sociales como aliados naturales y comenzaban a utilizar su preparación como la de especialistas en integración social. Mary Richmond se inventó el nombre de «Home Service» para designar este trabajo a escala nacional dirigió una monografía de treinta y seis páginas con este título y la publicó Cruz Roja ${ }^{14}$. Veía la oportunidad de exponer, con un lenguaje sencillo y ante una gran audiencia, los principios esenciales y los métodos del case work.

Alcanzada la paz, dado que la organización en que trabajaba estaba relacionada con la Cruz Roja, algunos de sus dirigentes se apresuraron a desarrollar un ambicioso programa social y sanitario, al que ella se opuso por dos motivos: por una parte, consideraba que era una violación de los acuerdos tácitos suscritos sobre el préstamo temporal de los mejores agentes sociales para responder a las necesida- des de urgencia y, por otra, porque consideraba inadecuado un proyecto de organización centralizada y centralizadora del Trabajo Social. Esta controversia nunca trascendió al debate público, pero sancionó la separación entre quienes pensaban que la Cruz Roja debía mantenerse como una organización humanitaria, circunscrita al marco de las situaciones de urgencia y a los desastres, y quienes querían continuar brindándole un espacio importante dentro de la práctica cotidiana del Trabajo Social.

\subsection{Profesora e investigadora de Trabajo Social}

Mary Richmond continuó infatigable con el proceso de elaboración del Case Work, enseñaba también de los trabajadores sociales, jóvenes y mayores, así como a los asistentes de los secretarios generales de las asociaciones. Participó activamente, desde 1905, en diferentes conferencias nacionales de caridad y rehabilitación (Havane, Portland, Filadelfia), convirtiéndose en una figura internacional. Creó una conferencia anual, daba cursos en la Escuela de Filantropía Aplicada de Nueva York e influyó indirectamente en la creación de la Escuela de Servicio Social y de la Salud de la Universidad de Pennsylvania.

Creó la supervisión en 1911, a la que le atribuía tres funciones principales: educativas, administrativas y de apoyo, y creó la conferencia de supervisión. De 1910 a 1922 desarrolló y dirigió varios institutos de verano, con la asistencia de las secretarias de las asociaciones y dirigió también cursos de verano. Desde su estancia en Baltimore, en 1902, tenía in mente escribir una obra para lo que había comenzado a recopilar material e informes sobre las familias que recibían ayuda, elaborados por los trabajadores sociales y cincuenta y siete agencias. A finales de 1902, había recogido tres cuartas partes de las «situaciones sociales» y las empezaba a analizar. En 1913, comenzó la redacción de este voluminoso estudio basado en 2.800 casos. La concepción del libro iba evolucionando y realizaba una constante revisión del plan de trabajo proyectado (Mary Richmond, descubrió la importancia de la revisión histórica y recurrió a los consejos de Miss Lucy Salomon,

${ }^{14}$ Cf. También de Mary Richmond (1913). War and Familly solidarity. Chesterton: Nabu Press. 
profesora de historia del Colegio Vassar). Inicialmente había pensado titular esta obra, First Steps in Treatement, pero luego, en 1916, eligió el título definitivo, Diagnóstico social, y el libro salió a la venta en 1917. Esta obra se considera un clásico ineludible de técnica profesional y se convirtió en la biblia de todo trabajador social. «Es cierto que ningún otro trabajo sobre servicio social había suscitado el respeto de otras profesiones como Social Diagnosis ${ }^{15}$. Más que un libro es el punto de anclaje del reconocimiento profesional del Trabajo Social.

Sin embargo Mary Richmond lo consideraba sólo el comienzo y quería que le siguiese otra obra. Después de la publicación de Diagnóstico social comenzó otro libro destinado a los voluntarios, en el que les trasmitía un acercamiento al social case work $^{16}$. Esta obra avanzaba muy lentamente y modificaba su proyecto en 1919, debido a los cambios sociales que se estaban produciendo. Por una parte, el estatuto de los voluntarios se estaba deteriorando y, por la otra, se profundizaba en la teoría del desarrollo de la personalidad. Este fenómeno iba a modificar su enfoque y decidió mostrar situaciones y métodos más complejos, tomando como apoyo los puntos fuertes del «cliente» y aplicándolos a todas las personas en situación de dificultad. Defendía la idea de que el de la asistencia material y el del case work eran dos enfoques diferentes, y que no tienen porqué ir asociados necesariamente ${ }^{17}$. También establecía una distinción entre el trabajador social, al que consideraba portador de una serie de competencias preciosas y una formación en el desarrollo de las relaciones humanas, y el individuo común, útil o personarecurso, preocupado por el Otro, pero sin competencias especiales ni una formación en este campo. Su enfoque promueve el desarrollo de la persona, de la familia y de su comunidad y, según la autora, se caracterizaba por: «los procesos que se desarrollan gracias a los ajustes aplicados a la personalidad consciente, individuo por individuo, entre los Hombres y su entorno social».

Como lectora y autora estaba muy preocupada por encontrar el término exacto y luchaba contra la jerga profesional del Trabajo Social, tratando de sustituirla por un vocabulario común, y modificaba continuamente los términos que creía obsoletos. Por ejemplo, hizo una elocuente defensa de la «National Conference of Social Workers», al rechazar denominaciones como «Social Welfare» o «Social Service» y también «Betterment Social».

En 1918, creó un grupo de trabajadores sociales que representaban los diferentes campos del Trabajo Social, que constituiría el comité de la organización profesional ${ }^{18}$, con dos objetivos: desarrollar una terminología y establecer un código ético.

$\mathrm{Su}$ salud comenzaba a deteriorarse y tenía que pasar los veranos en New Hampshire, donde el clima era más favorable y en donde, a partir de 1920, residirá regularmente. Aquí formaba parte de un grupo compuesto por universitarios y algunos amigos, era feliz de participar de esta nueva vida comunitaria, le encantaban los pic-nic, pero sobre todo los domingos por la tarde, cuando se organizaban debates espontáneos.

En 1921 tuvo la alegría de recibir, a título honorífico, el diploma del Master of Arts del Smith College: «por la elaboración de la base científica de una nueva profesión». Al año siguiente publicaba su obra What is Social Work? y abandonaba la Charity Russell Foundation de Nueva York.

A partir de 1924 su estado de salud empeoró, cada vez trabajaba más en casa, redujo sus contactos profesionales a algunos encuentros con jóvenes trabajadores sociales, que la con-

${ }^{15}$ Cf. The long vie (1930). Filadelfia: Russell Sage Foundation.

16 Este libro se tituló What is Social Case Work? Fue publicado en 1922.

17 Aunque apreciaba la teoría de Carl Jung (1875-1926), fundador de una corriente psicológica analítica, y la de Adolf Meyer (1866-1950), considerado el fundador de la psiquiatría americana, sin embargo ella siempre estimó y sostuvo que el rol más importante del Trabajo Social era el tratamiento individual, de la familia, y del entorno social

18 Este comité existió hasta la creación de la American Association of Social Workers en 1921. Mary Richmond mantuvo siempre mucho interés por el desarrollo de la organización profesional, pero murió sin haber sido jamás elegida por sus colegas presidenta de la Conferencia, a pesar de que se había postulado como candidata, pero era consciente de que existía una corriente de oposición a sus ideas. 
sideraban una leyenda. Organizó su vida en torno a las reuniones con sus viejos amigos, «The Talkers», en las que se trataban diversos temas como: métodos, historia, la nueva biología, la vida y los trabajos de Faraday y Pupin, la teoría electrodinámica, etc. y se apasionaba por las biografías...

$\mathrm{Su}$ última tarea fue proponer y trabajar para que se celebrara el quincuagésimo aniversario de la Charity Organisation de Estados Unidos, y aunque ya estaba casi impedida, pronunció una conferencia y recibió una calurosa ovación, en agradecimiento al esfuerzo realizado. Fue su último reencuentro con los trabajadores sociales. Al año siguiente se le diagnosticaba un tumor maligno, sin posibilidad de operación. Se internó en una residencia en Brighton, Massachusetts, y se dispuso a morir en paz. Allí corrigió las pruebas de un libro que no vio la luz (Marriage and the State, que fue publicado en 1929 tras una revisión del último capítulo). Murió en su casa de Nueva York, el 12 de Septiembre de 1928.

A modo de conclusión, se puede decir que la filantropía científica de Mary Richmond, la práctica de sus intervenciones cuyo resultado es el Case Work, su talento administrativo, su pasión por la investigación y su tenacidad en la formación del Trabajo Social han sido reconocidas de manera unánime.

\section{Referencias bibliográficas de Mary E. Richmond}

(1899). Friendy Visiting Hmong the Poor. Nueva York: The Macmillan Co,

(1907). The Good Neighbor in the Modern City. Philadelphia: J.B. Lippincott Co.

(1913). A study of nine hundred and eighty-five widows Known to certain charity organization societies en 1910. Nueva York: Russell Sage Foundation.

(1917) Social Diagnosis. Nueva York: Russell Sage Fondation.

(1922). What is Social Case Work? Nueva York: Russell Sage Foundation.

(1982). The biography of a social worker. Nueva York: American Association for organizing Famille Social Work.

Richmond, Mary E. y Sage Hall, Fred (1925). Child marriges. Nueva York: Russell Foundation.

Richmond, Mary E. y Sage Hall, Fred (1929). Marige and the State. Nueva York: Russell Foundation

Richmond, Mary E. y Sage Hall, Fred (1930). Tee long view. Nueva York: Russell Foundation (edic.póstuma)

\section{Referencias bibliográficas sobre Mary Richmond}

Agnew, Elisabeth (1924). From Charity to Social Work: Mary E. Richmond the creation of a new profession. Illinois: Universidad de Illinois.

Bouquet, Brigitte (2002). Prefacio para la reedición francesa del libro Les méhtodes nouvelles d'assitance. Le service sociale des cas individuels. Mary Richmond. Paris: Escuela de Altos Estudios de Salud Pública (EHESP)

Byington, Margaret Frances (1912). What socials workers should Know abaut their communitites an outline. Nueva York: Russell Sage Foundation, $2^{\mathrm{a}}$ ed. revisada y aumentada.

Kellogg, Marjorie (1951). The long journey: a biography of Mary Richmond, memorie. Northampton: Smith College School for Social Work.

Kirks-Adams, Karem (2009). Introduction to Social Work \& Social Welfare: Critical Thinking Perspectives., Belmont: Wadsworth Publishing Co. $3^{\text {a }}$ ed.

Mizrahi Terry, David Larry (2010). The enciclopedia social work. (4). Oxford: Oxford University Press.

Murdoch D'Alison, D. (1961). Mary Richmond, la professional. En Review social casework.

Specht, Harry y Courntney, Mark (1995). Unfaithful Angels: How Social Work Has Abonded Ists Mission. Engmand: The Free Press.

Stuart, Paul H. (1999). Linking clients and the police: social work's distinctive contribution. En Social Work 4, (44), 335-347. 